\title{
PERCEPÇÃO DAS MÃES FRENTE AO DIAGNÓSTICO DO FILHO COM DIABETES MELLITUS TIPO 1
}

\author{
Ilana Vanina Bezerra de Souza ${ }^{1}$, Daniela Karina Antão Marques ${ }^{2}$, Oneide Raianny Monteiro Lacerda ${ }^{3}$, Neusa Collet ${ }^{4}$
}

RESUMO: A criança/adolescente com Diabetes Mellitus requer apoio, compreensão, confiança e proteção da mãe, o que facilita a adesão ao tratamento, contribuindo para a qualidade de vida durante a evolução da doença. Este estudo teve como objetivo verificar a percepção das mães frente ao diagnóstico de diabetes do filho. A metodologia utilizada foi exploratória, descritiva e qualitativa, empregando a técnica do Discurso do Sujeito Coletivo. A análise evidenciou que as mães apresentam sentimentos oscilantes de luto durante as crises e, no momento da descoberta do diagnóstico, entram em desespero. A preocupação das mães refere-se às ocorrências constantes de descompensação, que traduzem a falta de equilíbrio da situação metabólica, gerando maior possibilidade do filho desenvolver complicações. A busca de informações e adaptação da rotina familiar visam proporcionar melhor qualidade de vida à criança/adolescente.

PALAVRAS-CHAVE: Mães; Diabetes mellitus; Criança; Adolescente; Doença crônica.

\section{MOTHERS' PERCEPTION OF THEIR CHILDREN'S DIAGNOSIS WITH TYPE ONE DIABETES MELLITUS.}

\begin{abstract}
A child with Diabetes Mellitus needs their mother's support, comprehension, confidence and protection, which facilitates compliance with treatment, contributing to quality of life during the progression of the illness. This study aimed to examine how a mother reacts when confronted with her child's diagnosis. The methodology used was exploratory, descriptive and qualitative, using the technique of Collective Subject Discourse. Analysis showed that mothers show varying feelings of bereavement during the crises and, at the time of initial diagnosis, fall into despair. The worry mothers feel is linked with the constant decompensation episodes, which manifest the instability of the child's metabolic situation, creating a greater risk of her developing complications. The search for information and adaptation in the family routine aim to improve the child/adolescent's quality of life.
\end{abstract}

KEYWORDS: Mothers; Diabetes mellitus; Child; Adolescent; Chronic illness.

\section{PERCEPCIÓN DE LAS MADRES DELANTE DEL DIAGNÓSTICO DEL HIJO CON DIABETES MELLITUS TIPO 1}

\begin{abstract}
RESUMEN: El niño/adolescente con Diabetes Mellitus nesecita apoyo, comprensión, confianza y protección de la madre, lo que facilita la adhesión al tratamiento, contribuyendo para la cualidad de vida durante la evolución de la enfermedad. Este estudio tuvo el objetivo de verificar la percepción de las madres delante del diagnóstico de diabetes del hijo. La metodología utilizada fue exploratoria, descriptiva y cualitativa, utilizándose la técnica del Discurso del Sujeto Colectivo. El análisis evidenció que las madres presentan sentimientos oscilantes de luto durante las crisis y, en el momento en que se descubrió el diagnóstico, entran en desesperanza. La preocupación de las madres se refiere a las ocurrencias constantes de descompensación, que traducen la falta de equilibrio de la situación metabólica, causando mayor posibilidad del hijo desarrollar complicaciones. La búsqueda de informaciones y adaptación de la rutina familiar tienen el propósito de proporcionar mejor cualidade de vida al niño/adolescente.
\end{abstract}

PALABRAS CLAVE: Madres; Diabetes mellitus; Niño; Adolescente; Enfermedad crónica.

\footnotetext{
${ }^{1}$ Enfermeira. Especialista em Metodologia do Ensino Superior. Professora da Faculdade de Enfermagem Nova Esperança-FACENE. ${ }^{2}$ Enfermeira. Mestre em Enfermagem. Enfermeira do Hospital Universitário Lauro Wanderley-UFPB. Professora da FACENE. ${ }^{3}$ Enfermeira. Mestranda no Programa de Ciências da Educação da Universidade Lusófona de Humanidades e Tecnologias. Professora da FACENE.

${ }^{4}$ Enfermeira. Doutora em Enfermagem. Professora do Curso de Graduação em Enfermagem e do Programa de Pós-Graduação em Enfermagem da UFPB.
}

\author{
Autor correspondente: \\ Daniela Karina Antão Marques \\ Universidade Federal da Paraíba \\ R. José Francisco da Silva, 1620 - 58071-120 - João Pessoa-PB-Brasil \\ E-mail: danielaantao@hotmail.com
}

Recebido: $01 / 09 / 10$

Aprovado: $21 / 12 / 10$ 


\section{INTRODUÇÃO}

Atualmente, no Brasil e no mundo, vive-se uma fase de mudança denominada transição epidemiológica, que consiste no aumento das doenças crônicas não transmissíveis (DCNT), entre elas o Diabetes Mellitus (DM). A importância dada a essa doença tem como fatores condicionantes o número crescente de portadores, a morte prematura, a incapacitação e os custos envolvidos no seu controle e no tratamento de suas complicações. Como entidade nosológica, situa-se entre as grandes preocupações da Organização Mundial de Saúde e é a terceira causa de morte no mundo ${ }^{(1)}$.

Quando afeta a criança/adolescente, o impacto do diagnóstico sobre a família envolve aspectos sociais, econômicos, afetivos e comportamentais, principalmente pela existência de complicações inerentes à patologia. Portanto, a família está sujeita à ansiedade, medos e expectativas de recuperação caracterizadas por um período de desestruturação e incertezas. É preciso que os familiares aprendam a lidar com sintomas e procedimentos terapêuticos e a ter habilidade para o aprendizado do controle e manejo da doença ${ }^{(3)}$.

O suporte familiar é fundamental para direcionar ações adequadas para a saúde, influenciando nos modos de adaptação necessários para garantir suporte ao paciente. $\mathrm{O}$ apoio por parte da família pode facilitar a adesão ao tratamento e contribuir para a prevenção de complicações em longo prazo, proporcionando melhoria da qualidade de vida durante a evolução do diabetes ${ }^{(4)}$.

Os profissionais de saúde e as famílias precisam unir esforços para que os portadores atinjam um bom controle metabólico, a fim de minimizar as complicações. Esses esforços devem ser direcionados para a manutenção dos níveis de glicose sanguínea dentro dos limites de normalidade, o que repercute na melhoria da qualidade de vida desses indivíduos ${ }^{(5)}$.

Com o intuito de motivar práticas cuidativas, é necessário compreender as mudanças que o DM desencadeia na vida da criança/adolescente e sua família, bem como o modo como se dão as demandas por busca de cuidado em saúde. Assim, a perspectiva da integralidade nos convida a repensar os modos como estão organizadas as práticas de cuidado destinadas à pessoa que vivencia essa condição crônica, com o intuito de responder as suas necessidades de saúde, que se expressam de maneira intensa no seu cotidiano ${ }^{(6)}$.

Famílias de crianças/adolescentes portadores de DM, muitas vezes, sentem-se impotentes no processo de convivência, devido à demanda por cuidados específicos, às limitações e ao enfrentamento da nova situação, a mudanças no estilo de vida e ao tratamento que a doença exige. Diante dessas dificuldades, os profissionais de saúde passam a intervir e a ter novas responsabilidades para contribuir no processo de adaptação e, pela adoção de estratégias, de modo a proporcionar às famílias o desenvolvimento de habilidades e conhecimentos voltados para a motivação do tratamento. E, ainda, para a reorganização familiar, por meio de informações sobre a doença e sua terapêutica ${ }^{(7)}$.

Ajudar os pais a reconhecer e responder aos comportamentos de seus filhos é importante, no sentido de promover e facilitar a interação do portador da doença com a sua família, ao permitir a participação nos cuidados com a criança/adolescente, além de fornecer apoio e aconchego após intervenção de rotina. Isso influencia o desenvolvimento do doente e facilita a formação e o fortalecimento do vínculo familiar ${ }^{(8)}$.

Considerando-se que, na constituição da família, o cuidado direto com a criança/adolescente, na maioria das vezes, é desempenhado pela mãe, esta pesquisa teve como objetivo verificar a percepção das mães frente ao diagnóstico do filho com Diabetes Mellitus tipo I.

\section{METODOLOGIA}

Trata-se de uma pesquisa exploratória e descritiva com abordagem qualitativa realizada em um Centro Especializado de Atenção Médica, do Município de João Pessoa/PB. O referido centro foi escolhido porque possui um serviço especializado no atendimento de pessoas com DM e por ter grande parte da demanda para essa modalidade de tratamento no Estado.

A amostra do estudo foi composta por 10 mães de crianças/adolescentes portadoras de DM tipo 1, que frequentaram o Centro no período da coleta de dados - outubro e novembro 2008 - e que atenderam aos critérios de seleção: ser mãe de criança/ adolescente com Diabetes Mellitus tipo 1; ser maior de idade; concordar e formalizar a participação na pesquisa; estar consciente e orientada no tempo e 
no espaço.

Para a produção do material empírico foi utilizada a técnica de entrevista semiestruturada. Trata-se de uma técnica que não é livre de estrutura, garantindo que dados coletados sejam adequados à questão da pesquisa, mas fornece ao entrevistador a oportunidade de aprofundamento sobre o assunto à medida que surgem as questões ${ }^{(9)}$.

O roteiro de entrevista foi composto por questões abertas, podendo o entrevistador esclarecer o significado das perguntas. Foi explanado o objetivo do estudo, a importância da participação da mãe, seguido da apresentação do Termo de Consentimento Livre e Esclarecido. A pesquisa foi realizada levando-se em conta os aspectos éticos em pesquisa envolvendo seres humanos e aprovada pelo Comitê de Ética em Pesquisa da Universidade Federal da Paraíba, sob protocolo no 106/08.

As questões relacionadas ao conhecimento acerca do DM foram analisadas pelo método qualitativo. Para essa análise, foi empregada a técnica do Discurso do Sujeito Coletivo (DSC), um conjunto de procedimentos que retratam as expressões-chave das falas dos pesquisados, o que viabiliza o pensamento em forma de síntese e possibilita a interpretação para fundamentação dos resultados ${ }^{(10)}$.

O DSC tem depoimentos como matéria-prima, sob a forma de um ou vários discursos-síntese escritos na primeira pessoa do singular. Visa expressar o pensamento de uma coletividade, como se esta coletividade fosse o emissor de um discurso ${ }^{(10)}$.

A técnica do DSC consiste em selecionar, de cada resposta individual dada a uma questão, as expressões-chave, que são trechos mais significativos destas respostas. A essas expressões correspondem Ideias Centrais, que são a síntese do conteúdo discursivo manifestado nas expressões-chave. Com o material das Expressões-Chave das Ideias Centrais constroem-se discursos-síntese, na primeira pessoa do singular, que são os DSC, nos quais o pensamento de um grupo ou coletividade aparece como se fosse um discurso individual ${ }^{(10)}$.

\section{RESULTADOS}

Os resultados analisados e apresentados no quadro 1 a seguir sintetizam as respostas obtidas a partir de dos dois questionamentos, empregando-se a técnica do Discurso do Sujeito Coletivo para cada uma das questões.
Quadro 1 - Ideia Central (IC) e Discurso do Sujeito Coletivo (DSC) composto por mães de filhos portadores de Diabetes Mellitus tipo 1. João Pessoa, 2008

Questionamento 1: Há quanto tempo a sra. sabe que seu filho é portador de Diabetes Mellitus tipo 1?

\section{IC 1 DSC}

Recentemente, [...] um mês descobri essa tristeza [...] desabafos vinte e cinco dias foi descoberta essa tristeza.

\section{IC 2 DSC}

Alguns meses [...] quatro meses minha única filha tem da descoberta essadoença [...]; tem oito meses [...]; faz seis meses [...]; cinco meses [...]; quatro meses que descobri essa doença.

Questionamento 2: Qual a sua percepção ao saber que seu filho é portador de Diabetes Mellitus tipo 1?

\begin{tabular}{ll}
\hline IC 1 & DSC \\
Desespero, uma & {$[\ldots]$ desespero por ser uma doença in- } \\
doença incurá- & curável [...]. Tive que me adaptar com \\
vel, não acre- & as mudanças, mas já conhecia mãe \\
dito & que tem essa doença [...]. Desespero \\
& por ser uma doença incurável, tive que \\
& me adaptar, pois... e agora? A minha \\
& única filha portadora, mas conheço a \\
& doença, minha mãe tem [...]. Chorei \\
& muito, desespero.
\end{tabular}

IC $2 \quad$ DSC

Muito difícil, [...] não me senti bem, né, porque é um principalmente jovem, eu pensei "como vou conseguir com um jovem controlar?", é dificil demais.

IC 3 DSC

Angustiante; [...] senti mal, angustiada. Não conhenão conhecia ço sobre a doença. Pensei que essa nada sobre a doença só dava em idoso [...]. Não doença acredito, não entendo, não conheço nada sobre a doença; ninguém da família tem esse problema [...]. Não conhecia nada.

IC 4 DSC

Com a hospita- [...] chorei muito, fiquei desesperada; lização, muito não sabia o que fazer, não encontrava choro ao saber vaga no hospital. Minha filha morrenque a filha é do- do e eu não sabia o que tinha. Quando ente foi hospitalizada e atendida pelo médico, ele me disse "ela é diabética". Fiquei desesperada, eu não sabia que doença era essa. 


\section{DISCUSSÃO}

A partir do Questionamento 1, IC1, evidencia-se que o impacto maior é o emocional ao descobrir a doença do filho, pois é nas crianças que os pais projetam sonhos e expectativas. As mães apresentam sentimentos oscilantes de luto, durante as crises dos filhos. Esse luto decorre do fato de não terem gerado uma criança "perfeita", pela incerteza em relação à vida, à morte, ao futuro e ao impacto disso na rotina familiar. A família pode não saber como agir, tendo atitudes ora de aproximação com o doente, ora de isolamento emocional. A família pode isentar o doente de responsabilidades e atividades, isolando-o do convívio. A expectativa da perda está presente e é clara para todos os familiares, fazendo com que mobilizem recursos para lidar com isso.

Em relação ao paciente diabético, o suporte familiar é fundamental, pois é um aliado na aquisição de orientações de saúde adequadas e no processo de enfrentamento da doença. Assim, o processo educativo deve valorizar as experiências e os conhecimentos antecedentes, guiado por valores e crenças do próprio paciente e de seu núcleo familiar acerca da doença ${ }^{(3,}$ ${ }^{11-12)}$.

Já com relação à IC2, a literatura mostra que se a família tem conhecimento prévio e mais tempo de convivência com a doença, o tratamento e a aceitação se tornam mais fáceis e seus níveis de estresse e ansiedade podem diminuir significativamente. A família que apóia seus membros em situação de doença compreende as modificações relacionadas à condição e torna-se permeável aos ajustamentos necessários para garantir o suporte necessário ao seu familiar, facilitando a adesão ao tratamento, a recuperação e a melhora de sua saúde. Desse modo, podem ser asseguradas as condições para uma maior eficácia $\operatorname{adaptativa}^{(12-13)}$.

Quanto à percepção da mãe ao saber que seu filho é portador de DM tipo I, o Questionamento 2 mostra quatro ideias centrais: IC1 "Desespero, uma doença incurável, não acredito"; IC2 "Muito difícil, principalmente com um jovem"; IC3 "Angustiante, não conhecia nada sobre a doença"; IC4 "Não encontrava vaga nos hospitais; muito choro ao saber que minha filha é doente".

A IC1 evidencia que as mães, no momento da descoberta do diagnóstico, entram em desespero e, de acordo com a literatura, o sentimento mais comum nesse momento inicial de descoberta da doença é o de negação. A negação ocorre mais no início do processo da doença crônica, mas pode recorrer em outros momentos ${ }^{(11,14)}$.

A existência de uma doença crônica afeta toda a família e gera momentos difíceis, com avanços e retrocessos nas relações entre seus membros. O cuidado à criança com doença crônica consome energia, tempo, retira a privacidade da família e pode provocar isolamento social e emocional. As limitações experenciadas pela criança com diabetes são inúmeras e desencadeiam vários sentimentos, como o medo, insegurança e atitudes que vão do conformismo ao autocuidado $^{(11-12,14)}$. Todos esses sentimentos são vivenciados por um longo período de tempo. Além disso, as emoções geradas na criança diante do enfrentamento da doença crônica são semelhantes às emoções vivenciadas por qualquer faixa etária, como a negação, a minimização da doença, a raiva e a frustração pela limitação da doença, sintomas depressivos, culpa, procura de soluções impossíveis, entre outras ${ }^{(11-12,14)}$.

Os resultados da IC2 evidenciam, de acordo com as mães, que a criança enfrenta barreiras e limitações referentes à dieta e à aplicação da insulina. Nessa fase, a mãe demonstra que a compreensão da doença ultrapassa os limites físicos e biológicos e busca em Deus - suporte religioso - respostas às suas questões, colocando-o como condutor de tudo que acontece na vida das pessoas. Sendo assim, tem que aceitar, pois acredita que Deus sabe o que faz e se decidiu por ela, é porque ela pode superar tudo isso.

A família, desde o início dos sintomas até a definição do diagnóstico e tratamento, vivencia uma fase de crise, caracterizada por um período de desestruturação e incertezas, tendo que aprender a lidar com sintomas, procedimentos diagnósticos e terapêuticos, para assim reorganizar suas vidas ${ }^{(12,14)}$.

Os resultados da IC3 evidenciam, de acordo com as entrevistadas, que elas não conheciam a doença, porém tentaram aprender sobre o assunto por meio de orientações profissionais, livros, jornais e internet, com o objetivo de cuidar melhor de seu filho.

A literatura mostra que a organização familiar influencia fortemente o aprendizado da família ${ }^{(12,15)}$. Portanto, a participação familiar no processo educativo contribui para o seguimento do tratamento, na medida em que serve como fonte de apoio emocional nos momentos em que o paciente se sente impotente diante dos desafios advindos da doença. A informação, tanto para a família quanto para a criança, é um meio eficaz de minimizar os sentimentos de incerteza, 
medo, dor e desconforto inerentes ao diagnóstico de uma condição mórbida para a qual não se tem a perspectiva da cura, mas tão somente do controle clínico $^{(11,15-16)}$.

Para o controle da doença, o apoio da família e dos amigos é fundamental, e estudos mostram que pacientes que tiveram apoio adequado de amigos e familiares aderiram melhor às condutas do autocuida$\mathrm{do}^{(12-13,17)}$. Avaliar os meios de apoio do paciente pode ajudar a identificar as suas necessidades de assistência e evitar complicações a longo prazo ${ }^{(13,17)}$.

O resultado da IC4 evidenciou que as mães se sentem angustiadas pela hospitalização e desesperadas por não saber o que o filho tem. As mães de crianças portadoras de doença crônica ficam expostas a uma série de eventos que desencadeiam medo, angústia e insegurança. Elas sofrem por não saber o que pode acontecer ao filho, pelas incertezas quanto à doença e ao tratamento, por temer a possibilidade de algo dar errado e pelo medo de perder o seu filho. A mãe sofre assistindo ao sofrimento, sofre junto com o filho e sofre pelo fato de vê-lo sofrendo, especialmente nas situações em que é submetido a procedimentos terapêuticos dolorosos, sem que ela possa fazer alguma coisa para evitar ou minimizar a dor. A mãe também vivencia a dor de sentir-se impotente, de ser incapaz de livrar o seu filho do sofrimento ${ }^{(18)}$.

Além das alterações estabelecidas pela doença para a criança em relação à alimentação, inserção de exercícios físicos no cotidiano e insulinoterapia, as mães acrescentam à experiência que seus filhos vivenciam ao se sentirem diferentes de seus colegas, por não terem a mesma liberdade que eles, devido aos horários e restrições impostas pelo tratamento. A preocupação das mães refere-se às ocorrências constantes de descompensação, que traduzem a falta de equilíbrio da situação metabólica, gerando maior possibilidade de a criança vir a desenvolver complicações, o que compromete sua qualidade de vida no futuro.

\section{CONSIDERAÇÕES FINAIS}

A confirmação do diagnóstico de Diabetes Mellitus tipo I é um marco profundo na vida da família, dividindo-a em "antes" e "depois" da descoberta da doença da criança. A vida da família passa a ser influenciada pela doença do filho e o relacionamento entre os membros desta família também pode sofrer alterações.
Este estudo possibilitou ampliar o conhecimento sobre a percepção das mães frente ao diagnóstico do filho com Diabetes Mellitus tipo 1, evidenciando as dificuldades enfrentadas, principalmente no momento da descoberta da doença. A confirmação do diagnóstico implica no estabelecimento de uma nova rotina para o sistema familiar. Contudo, o enfrentamento positivo de algumas famílias pode ser encontrado a partir do momento em que houve a busca de informações e a tentativa de adaptação da rotina familiar, visando proporcionar melhor qualidade de vida ao filho.

A informação adequada e compreensível, por meio da educação em saúde, é destaque relevante em relação à problemática deste estudo. Observou-se que ela contribui diretamente no fortalecimento da mãe, que se sente apoiada, valorizada e estimulada a cuidar do filho. A mãe prossegue manifestando sua satisfação com a qualidade das informações fornecidas pelos profissionais de saúde e se mostra bem informada sobre a doença.

Destacamos o potencial e contribuições do enfermeiro, neste contexto, como educador para ações de prevenção de agravo e de cuidado.

\section{REFERENCIAS}

1. Nunes MDR, Dupas G, Ferreira NMLA. Diabetes na infância/adolescência: conhecendo a dinâmica familiar. Rev Eletr Enf. [Internet] 2007;9(1) [acesso em 10 mai 2010]. Disponível: http://www.fen.ufg.br/ revista/v9/n1/v9nla09.htm

2. Ministério da Saúde (BR). Diabetes mellitus. Brasília: Ministério da Saúde; 2005.

3. Dias DG, Santana MG, Santos E. Percebendo o ser humano diabético frente ao cuidado humanizado. Rev Bras Enferm. 2006;59(2):168-71.

4. Zanetti ML, Biagg MV, Santos MA, Péres DS, Teixeira CRS. O cuidado à pessoa diabética e as repercussões na família. Rev Bras Enferm. 2008;61(2):186-92.

5. Péres DS, Santos MA, Zanetti ML, Ferronato AA. Dificuldade dos pacientes diabéticos para controle da doença: sentimentos e comportamentos. Rev LatinoAm Enfermagem. 2007;15(6):1105-12.

6. Thaines GHLS, Bellato R, Faria APS, Araújo LFS. A busca por cuidado empreendida por usuário com diabetes mellitus: um convite à reflexão sobre a integralidade em saúde. Texto \& Contexto Enferm. 
2009;18(1):57-66.

7. Mello MB. Déficits de autocuidado vivenciados por famílias de crianças e adolescentes com diabetes mellitus tipo 1 [dissertação]. Florianópolis (SC): Universidade Federal de Santa Catarina; 2006.

8. Doré JS, Imamura PEA, Figueira BBD, Fernandes GCV. Humanização e abordagem desenvolvimentista nos cuidados ao recém-nascido de muito baixo peso. São Paulo: Atheneu; 2003.

9. Meyer P. Tipos de coletas de dados na pesquisa qualitativa. In: Pope C, Mays N. Pesquisa qualitativa na atenção à saúde. $2^{\mathrm{a}}$ ed. Porto Alegre: Artmed; 2005. p. $12-21$.

10. Lefèvre F, Lefèvre AM. C. O discurso de sujeito coletivo: um novo enfoque em pesquisa qualitativa (desdobramento). Caxias do Sul: Educs; 2005.

11. Pilger C, Abreu IS. Diabetes mellitus na infância: repercussões no cotidiano da criança e de sua família. Cogitare Enferm. 2007;12(4):494-501.

12. Moreira PL, Dupas G. Vivendo com diabetes: a experiência contada pela criança. Rev Latino-Am Enfermagem. 2006;14(1):25-32.

13. Marcelino DB, Carvalho MDB. Reflexões sobre o diabetes tipo 1 e sua relação com o emocional. Psicol Reflex Crit. 2005;18(1):72-7.

14. Zanette ML, Mendes IAC, Ribeiro KP. O desafio para o controle domiciliar em crianças e adolescentes diabéticas tipo 1. Rev Latino-Am Enfermagem. 2001;9(4):32-6.

15. Santos ECB, Zanetti ML, Otero LM, Santos MA. O cuidado sob a ótica do paciente diabético e de seu principal cuidador. Rev Latino-Am Enfermagem. 2005;13(3):397-406.

16. Castro ARV, Grossi SAA. Custo do tratamento do diabetes mellitus tipo 1: dificuldades das famílias. Acta Paul Enferm. 2008;21(4):624-8.

17. Silveira AO, Ângelo M, Martins SR. Doença e hospitalização da criança: identificando as habilidades da família. Rev Enferm UERJ. 2008;16(2):212-7.

18. Oliveira I, Angelo M. Vivenciando com o filho uma passagem difícil e reveladora: a experiência da mãe acompanhante. Rev Esc Enferm USP. 2000;34(2):2028. 